\title{
Direct Spectroscopic Observation of a Shallow Hydrogenlike Donor State in Insulating $\mathrm{SrTiO}_{3}$
}

Z. Salman, ${ }^{*}$ T. Prokscha, ${ }^{\dagger}$ A. Amato, E. Morenzoni, R. Scheuermann, K. Sedlak, and A. Suter Paul Scherrer Institute, Laboratory for Muon Spin Spectroscopy, CH-5232 Villigen PSI, Switzerland (Received 9 May 2014; revised manuscript received 8 September 2014; published 7 October 2014)

We present a direct spectroscopic observation of a shallow hydrogenlike muonium state in $\mathrm{SrTiO}_{3}$ which confirms the theoretical prediction that interstitial hydrogen may act as a shallow donor in this material. The formation of this muonium state is temperature dependent and appears below $\sim 70 \mathrm{~K}$. From the temperature dependence we estimate an activation energy of $\sim 50 \mathrm{meV}$ in the bulk and $\sim 23 \mathrm{meV}$ near the free surface. The field and directional dependence of the muonium precession frequencies further supports the shallow impurity state with a rare example of a fully anisotropic hyperfine tensor. From these measurements we determine the strength of the hyperfine interaction and propose that the muon occupies an interstitial site near the face of the oxygen octahedron in $\mathrm{SrTiO}_{3}$. The observed shallow donor state provides new insight for tailoring the electronic and optical properties of $\mathrm{SrTiO}_{3}$-based oxide interface systems.

DOI: 10.1103/PhysRevLett.113.156801

PACS numbers: 73.21.-b, 36.10.Ee, 76.75.+i

The discovery of a high mobility two-dimensional electron gas (2DEG) at the interface between two insulating perovskite oxides, $\mathrm{TiO}_{2}$-terminated $\mathrm{SrTiO}_{3}$ (STO) and $\mathrm{LaAlO}_{3}$ (LAO) [1-3], has prompted great interest in these oxides. In addition to the 2DEG, this interface was found to be magnetic [4-6] and even superconducting below $\sim 300 \mathrm{mK}$ [7]. It is generally agreed that the high carrier densities at the interface are associated with various effects, including doping with electrons or oxygen vacancies [2,8-11], interdiffusion [10,12,13], and the influence of lattice distortions [14-18]. These discoveries provide an interesting prospect for producing interfaces with physical properties not present, nor predictable, from the constituent materials, and may lead to new technological applications. Here we discuss the response of these oxides to impurities, in particular hydrogen, which may play an important role in the discovered phenomena. Moreover, we note that the reported results are extremely relevant for possible applications of STO and related insulating oxides in fuel cells and hydrogen sensors [19-21].

Hydrogen is a ubiquitous impurity in device or sample fabrication and can cause unintended modifications of the electronic and structural properties. For example, the properties of the 2DEG at STO/LAO interfaces may be affected if additional free charge carriers are present due to unintentional doping [22]. The electronic behavior of interstitial hydrogen can be characterized by the position of the $\mathrm{H}(+/-)$ level in the band gap, where the formation energies of $\mathrm{H}^{+}$and $\mathrm{H}^{-}$are equal. If this level is close to or intersecting the conduction (valence) band, hydrogen will act as a shallow donor (acceptor). In elemental and binary semiconductors, a universal alignment of the $\mathrm{H}(+/-)$ level at $\sim-4.5 \mathrm{eV}$ with respect to the vacuum level has been found theoretically [23] and supported by experiments [24]. The model predicted successfully hydrogen shallow donor states in $\mathrm{ZnO}$ and $\mathrm{InN}$ [25-27]. In this model the $\mathrm{H}(+/-)$ level coincides approximately with the host's charge neutrality level (CNL). In general, the CNL is found to be located at relatively constant energies with respect to the vacuum level $[23,28]$. The coincidence of the $\mathrm{H}(+/-)$ level with the CNL can be understood as follows. In a binary semiconductor $\mathrm{H}^{+}$and $\mathrm{H}^{-}$behave similarly; i.e., they break a bond at the anion or cation site, respectively, leaving a dangling bond at the opposite cation or anion site. The $\mathrm{H}(+/-)$ level is then located midway in between the energy levels of the dangling bonds, which corresponds to the CNL.

In oxides the situation is different, since $\mathrm{H}^{+}$tends to form an $\mathrm{OH}^{-}$antibonding state, without breaking a cation$\mathrm{O}$ bond. $\mathrm{H}^{-}$, on the other hand, causes the formation of an oxygen dangling bond by breaking a metal-O bond. Thus, the $\mathrm{H}(+/-)$ level is determined by the average of the oxygen dangling bond and $\mathrm{OH}^{-}$antibonding levels, which is located at a relatively constant energy of about $4.5-5.5 \mathrm{eV}$ above the valence band maximum [28,29]. Therefore, hydrogen should form a shallow donor state in oxides with a band gap of less than $\sim 4.5 \mathrm{eV}$, such as STO which has an indirect band gap of $\sim 3.2 \mathrm{eV}$. This prediction has been supported recently by a new theoretical work on hydrogenated vacancies and hidden hydrogen in STO [30]. In contrast, the universal alignment model described above places $\mathrm{H}(+/-)$ deep in the band gap, i.e., $\sim 0.5 \mathrm{eV}$ below the conduction band minimum, which equals the electron affinity of STO and is located at about $4.0 \mathrm{eV}$ below the vacuum level. Note, the model in Refs. [28,29] predicts shallow donor states in $\mathrm{SnO}_{2}, \mathrm{TiO}_{2}, \mathrm{ZrO}_{2}$, and $\mathrm{HfO}_{2}$ which have been observed or inferred in muon spin rotation $(\mu \mathrm{SR})$ experiments [31]. In these experiments, positive muons, when implanted into insulators or semiconductors, can capture an electron to form interstitial muonium 
$\left[\mathrm{Mu}=\left(\mu^{+} \mathrm{e}^{-}\right)\right]$, which can be considered as a light hydrogen isotope and mimics its chemical and electrical interactions. In fact, a large amount of information on the structure and electrical activity of isolated interstitial $\mathrm{H}$ states in semiconductors and insulators has been obtained by $\mathrm{Mu}$ spectroscopy $[32,33]$.

In this Letter we present a direct spectroscopic observation of a shallow hydrogenlike Mu state in a STO (100) single crystal, in agreement with the model suggested by Refs. [28,29]. Our results are consistent with muons occupying an interstitial site in the lattice, between two $\mathrm{O}-\mathrm{O}$ bonds near the face of the oxygen octahedron. We find that up to $\sim 60 \%$ of the implanted muons form a shallow muonium state at $25 \mathrm{~K}$ with a relatively small hyperfine coupling. From the field dependence of the Mu characteristic precession frequencies we find that the hyperfine tensor is fully anisotropic and estimate the hyperfine interaction along the principal axes of the tensor $A_{X}=1.4 \pm 0.1 \mathrm{MHz}, A_{Y}=6.7 \pm 0.1 \mathrm{MHz}$ and $A_{Z}=$ $11.5 \pm 0.1 \mathrm{MHz}$. This $\mathrm{Mu}$ state is one of the rare clear examples of a fully anisotropic $\mathrm{Mu}$.

The $\mu \mathrm{SR}$ experiments were performed on the DOLLY, GPS, and LEM spectrometers at the Paul Scherrer Institut in Villigen, Switzerland. In these experiments $100 \%$ polarized positive muons are implanted into the sample. Each implanted muon decays (lifetime $\tau_{\mu}=2.2 \mu \mathrm{s}$ ) emitting a positron preferentially in the direction of its polarization at the time of decay. Using appropriately positioned detectors, one measures the asymmetry of the muon beta decay along different directions as a function of time, $A(t)$, which is proportional to the time evolution of the muon spin polarization. $A(t)$ depends on the electronic environment of the muon and is used to extract information on the hyperfine interaction between the muon and the electrons in the system. Muonium is spectroscopically identified by its characteristic precession frequencies which allow us to determine the $\mathrm{Mu}$ hyperfine parameters [32,33]. In a low energy $\mu \mathrm{SR}$ (LE- $\mu \mathrm{SR})$ experiment, the energy of the implanted muons, $E$, can be tuned $(1-30 \mathrm{keV})$ to perform a measurement of $A(t)$ at depths in the range $\sim 1-200 \mathrm{~nm}$ $[34,35]$. The measurements reported here were performed on a $15 \times 15 \times 1 \mathrm{~mm}$ single side epitaxially polished (100) STO single crystal substrate (Crystal $\mathrm{GmbH}$ ). In the bulk $\mu \mathrm{SR}$ measurements, the sample was suspended on an aluminized Mylar tape and mounted into a He gas flow cryostat. The muons were implanted with their polarization nominally along $\langle 100\rangle$ with the field applied perpendicular to it (nominally along $\langle 010\rangle$ ). In the LE- $\mu$ SR measurements the sample was glued to a cold finger cryostat, with the field applied along $\langle 100\rangle$ and the polarization of implanted muons perpendicular to it.

We start by looking at muon spin precession measurements in a field of $B=10 \mathrm{mT}$ applied perpendicular to the muon's initial spin direction. At room temperature we find that all muons implanted at $4.1 \mathrm{MeV}$ in bulk STO precess at

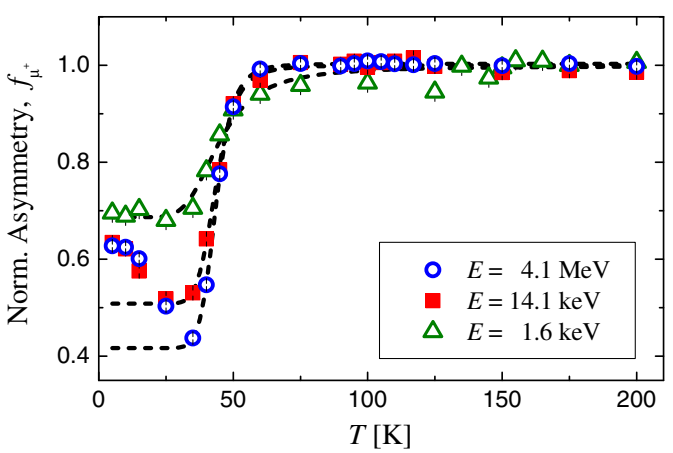

FIG. 1 (color online). The normalized diamagnetic fraction of the $\mu^{+}$precession signal at $B=10 \mathrm{mT}$ as a function of temperature. Circles, squares, and triangles are measurements at 4.1 MeV $(B \perp\langle 100\rangle), 14.1 \mathrm{keV}$, and $1.6 \mathrm{keV}(B \|\langle 100\rangle)$, respectively. The drop in asymmetry below $\sim 70 \mathrm{~K}$ is due to formation of muonium. The dashed lines are fits to Eq. (1).

the Larmor frequency of $\mu^{+}, \nu_{0}=\gamma_{\mu} / 2 \pi B$, with almost no damping. Here, $\gamma_{\mu} / 2 \pi=135.5 \mathrm{MHz} / \mathrm{T}$ is the muon gyromagnetic ratio. As we decrease the temperature, we find that below $\sim 70 \mathrm{~K}$ the amplitude of the signal precessing at $\nu_{0}$ (the so called diamagnetic fraction, $f_{\mu^{+}}$) decreases sharply, reaches a minimum at $\sim 30 \mathrm{~K}$, and then increases at lower temperatures (Fig. 1). Similar behavior is observed at lower muon implantation energies. However, in this case the decrease in the $f_{\mu^{+}}$is smaller, and at $E=1.6 \mathrm{keV}$ we observe no increase at low temperatures.

Closer investigation of the measured asymmetries in the bulk below $70 \mathrm{~K}$ reveals that the polarization contains additional components with precession frequencies higher than $\nu_{0}$. Example asymmetries, measured at $25 \mathrm{~K}$ and applied fields of 1 and $10 \mathrm{mT}$ are shown in Fig. 2(a). Note that the additional frequencies are of the order of few $\mathrm{MHz}$
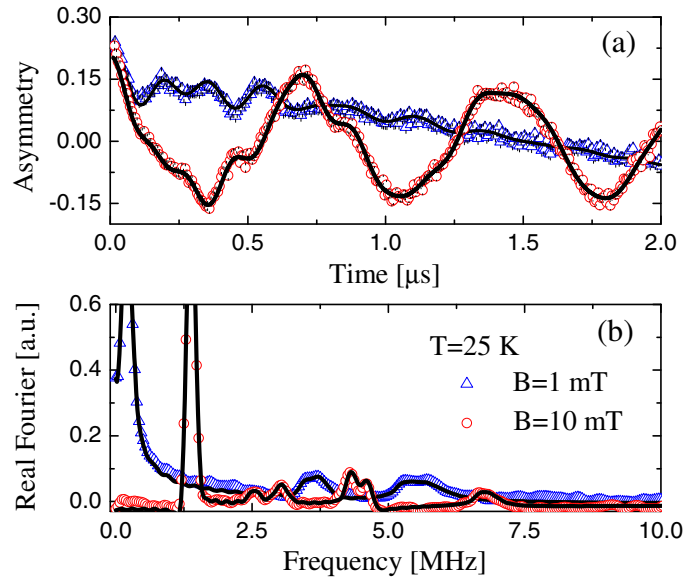

FIG. 2 (color online). (a) The asymmetries at $T=25 \mathrm{~K}$ as a function of time for applied fields 1 and $10 \mathrm{mT}(B \perp\langle 100\rangle)$ and (b) the corresponding real Fourier transform showing at least four muonium precession frequencies in both cases. The solid lines are fits to a sum of precessing and damping signals. 
[see Fig. 2(b)], which we attribute to $\mathrm{Mu}$ precession frequencies. These are directly related to the hyperfine interaction of $\mathrm{Mu}$ and confirm its formation at these temperatures. The size of these frequencies $(<10 \mathrm{MHz})$ indicates that a "shallow" $\mathrm{Mu}$ state is formed, i.e., much smaller than the $\sim 4.4 \mathrm{GHz}$ frequency observed for vacuum $\mathrm{Mu}$.

The temperature dependence of $f_{\mu^{+}}$is related to the $\mathrm{Mu}$ fraction, $f_{\mathrm{Mu}}=1-f_{\mu^{+}}$. It can be calculated following a semiempirical model [31],

$$
f_{\mu^{+}}=1-f_{\mathrm{Mu}}=1-\frac{f_{0}}{1+N \exp (-\Delta / T)},
$$

where $f_{0}$ is the normalized maximum $\mathrm{Mu}$ fraction, $N$ is a density-of-states parameter, and $\Delta$ is the Mu activation energy which can be considered as an effective ionization or binding energy [31]. A fit of $f_{\mu^{+}}$in bulk (circles in Fig. 1), between 25 and $200 \mathrm{~K}$, yields an ionization energy of $\Delta=600 \pm 15 \mathrm{~K}$ or $52 \pm 2 \mathrm{meV}$. Similar fits for the data at low implantation energies yield $\Delta=515 \pm 15 \mathrm{~K}$ $(45 \pm 2 \mathrm{meV})$ and $270 \pm 10 \mathrm{~K} \quad(23 \pm 1 \mathrm{meV})$ for $14.1 \mathrm{keV}$ and $1.6 \mathrm{keV}$, respectively. Similar decrease in shallow muonium ionization energy near the surface has been recently detected in $\mathrm{ZnO}$ and $\mathrm{CdS}$. This effect is attributed to the presence of electric fields due to band bending near the free surface [36].

It is worthwhile noting here that the absence of (or weak) energy dependence of the diamagnetic fraction below $\sim 10 \mathrm{~K}$ indicates that the $\mathrm{Mu}$ formation at low $T$ does not depend on the number of track electrons; i.e., there is no delayed $\mathrm{Mu}$ formation below $T \sim 10 \mathrm{~K}$. Mu can form in two ways: (i) promptly during charge-exchange collisions with subsequent thermalization as neutral $\mathrm{Mu}$, and (ii), delayed, where the positive muon thermalizes at an interstitial lattice site, followed by a capture of an electron from its own ionization track. The latter is significantly suppressed if the number of track electrons is $\lesssim 10^{3}$, corresponding to implantation energies of less than a few $\mathrm{keV}$ [37]. It is also suppressed in materials with a large dielectric constant, $\varepsilon$, where the electric field of the muon point charge is effectively screened by the surrounding medium, thus suppressing the probability of capturing a track electron. In STO, $\varepsilon \sim 300$ at room temperature, reaches $\sim 10000$ at $\sim 25 \mathrm{~K}$, and then saturates at 20000-25000 below $10 \mathrm{~K}$ [38]. The increase of the diamagnetic fraction below $\sim 30 \mathrm{~K}$ in the bulk may be due to the increasing $\varepsilon$ at these temperatures, as delayed $\mathrm{Mu}$ formation becomes less likely. The low temperature flattening of $f_{\mu^{+}}$corresponds well to the saturation of $\varepsilon$.

It is somewhat surprising that a shallow Mu state can be observed in a system with such a large $\varepsilon$. For shallow donors, one usually applies a hydrogenic effective-mass model to estimate the binding energy of the donor to be $E_{D} \sim R_{y}\left(m^{*} / m_{e}\right) / \varepsilon^{2}$, where $R_{y}=13.6 \mathrm{eV}$ the Rydberg constant, $m_{e}$ the electron mass, and $m^{*}$ the conductionband effective mass of the electron [31]. This approximation is justified by the large electron wave function spreading over several lattice sites. This implies that at $25 \mathrm{~K}$, where $\varepsilon \sim 10000$ in STO, the binding energy should be zero; i.e., no Mu should form. Therefore, the observation of a shallow $\mathrm{Mu}$ state indicates that it might have a more localized polaronic character [31]. Furthermore, it is known that doping of STO may increase $m^{*} / m_{e}$ up to 20 [39], and that an electric field may lower $\varepsilon$ [40]. Hence, "doping" STO with Mu may cause a local deformation of the lattice with a local modification of $m^{*}$ and $\varepsilon$.

Now we turn to evaluating the hyperfine interaction tensor of $\mathrm{Mu}$. We consider a general Hamiltonian for a muon (spin I) interacting with an electron $(\operatorname{spin} \mathbf{S})$ with a fully anisotropic hyperfine interaction [41],

$$
\mathcal{H}=\gamma_{e} B S_{z}-\gamma_{\mu} B I_{z}+A_{Z} S_{Z} I_{Z}+A_{Y} S_{Y} I_{Y}+A_{X} S_{X} I_{X} .
$$

where $\gamma_{\mu}$ and $\gamma_{e}$ are the muon and electron gyromagnetic ratios, $z$ is defined by the direction of $B$ and $A_{i}(i=X, Y, Z)$ are the $\mathrm{Mu}$ hyperfine interactions along the principal axes. The coordinates $(X, Y, Z) /(x, y, z)$ denote the hyperfine interaction or laboratory frame, such that the components of a spin angular momentum vector seen in the $(X, Y, Z)$ system are expressed in terms of the components of the same vector seen in the $(x, y, z)$ system as

$$
\left[\begin{array}{l}
S_{X} \\
S_{Y} \\
S_{Z}
\end{array}\right]=D(\alpha, \beta, \gamma)\left[\begin{array}{l}
S_{x} \\
S_{y} \\
S_{z}
\end{array}\right],
$$

where $\alpha, \beta$, and $\gamma$ are the Euler angles of the three consecutive rotations around $Z, Y$, and $Z$ axes of the $(X$, $Y, Z)$ coordinate system, which initially coincides with the $(x, y, z)$ system. In zero field (ZF), the Hamiltonian, Eq. (2), can be diagonalized analytically to calculate the $\mathrm{Mu}$ frequencies, giving a maximum of 6 possible frequencies depending on the values of $A_{i}$ [41]. These are the sums and differences of the different $A_{i}$ parameters. Indeed our ZF measurement, shown in Figs. 3(a) and 3(b), with different relative orientations between the implanted muon spin and STO crystal give $\nu=2.4,2.7,4.1,5.1,6.5$ and $9 \mathrm{MHz}$ [Figs. 3(c) and 3(d)]. From these we estimate the hyperfine parameters $A_{X}=1.4 \pm 0.1 \mathrm{MHz}, A_{Y}=6.7 \pm 0.1 \mathrm{MHz}$ and $A_{Z}=11.5 \pm 0.1 \mathrm{MHz}$.

Next, we extract the field dependent $\mathrm{Mu}$ precession frequencies from the asymmetries measured in bulk STO at $25 \mathrm{~K}$. Here, we limit ourselves to four or five most visible frequencies for each field, as plotted in Fig. 4. Using $A_{i}$ values we calculate the $\mathrm{Mu}$ frequencies in an applied field by numerical diagonalization of $\mathcal{H}$. The amplitudes or probabilities of precession between different $\mathrm{Mu}$ energy states depend on the initial spin direction of the muon 

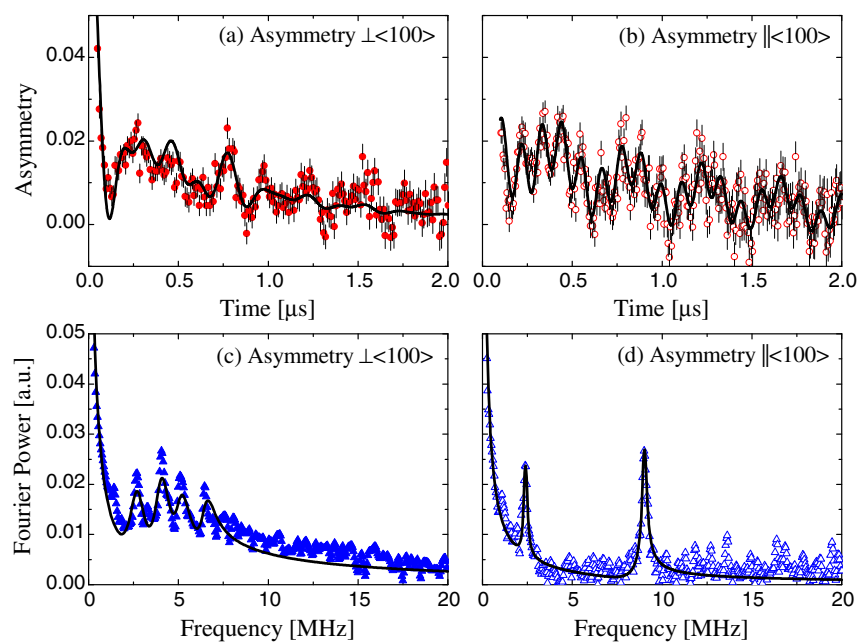

FIG. 3 (color online). The asymmetries measured (a) parallel and (b) perpendicular to $\langle 100\rangle$ at $T=25 \mathrm{~K}$ and in ZF. (c) and (d) are the corresponding Fourier transform showing clear ZF muonium precession frequencies in both cases. The solid lines are fits to a sum of precessing and damping signals

relative to the hyperfine principal axes. We find that in order to best model the field dependence in Fig. 4, one has to set the $(\alpha, \beta, \gamma)=\left(0^{\circ}, 57^{\circ}, 27^{\circ}\right)$ (solid lines in Fig. 4) and $\left(0^{\circ}, 17^{\circ}, 0^{\circ} \pm 45^{\circ}\right)$ (dashed lines in Fig. 4). The agreement with our experimental results is excellent considering the uncertainties and limited resolution in the experimental data. Note, there is a small frequency splitting in the highest frequency branch. This may be attributed to a small amount of mosaicity or the structural distortion in STO at this temperature [42]. Such effect results in different domain orientations and thus a small variation in the local environment of $\mathrm{Mu}$, which can only be resolved at high frequencies. Using the extracted hyperfine parameter to calculate the precessing amplitudes reveals that there is no missing fraction, i.e., $f_{\mu^{+}}+f_{\mathrm{Mu}}^{\mathrm{S}}=1$, where $f_{\mathrm{Mu}}^{\mathrm{S}}$ is the fraction of shallow $\mathrm{Mu}$. This excludes the existence of any Mu state deep in the band gap. Although we cannot exclude that the observed neutral muonium center is metastable, the fact that the sum of the $\mathrm{Mu}^{+}$and $\mathrm{Mu}^{0}$ fractions accounts for the full muon polarization, suggests an equilibrium balance between the two charge states. The observed hyperfine interaction strength and ionization energy imply a shallow donor state, modeling the analogous hydrogen state [43].

Note that the angle between the STO cubic axes and the normal to the face of the oxygen octahedron is $\sim 54^{\circ}$. Therefore, the hyperfine tensor and the first set of angles (solid lines in Fig. 4) could be attributed to a Mu occupying an interstitial site between two $\mathrm{O}-\mathrm{O}$ bonds and near the face of the oxygen octahedron in the STO crystal. The determination of the second set of angles (dashed lines in Fig. 4) is much less reliable since it shows a much weaker angular dependence. Nevertheless, if we assume that the angle between the applied field and the $\langle 010\rangle$ is $\sim 14^{\circ}$, then these

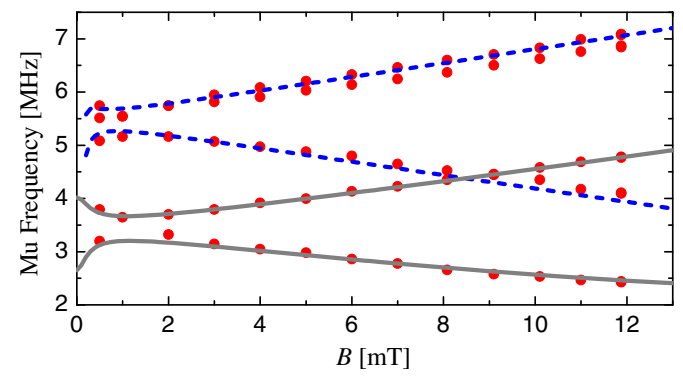

FIG. 4 (color online). The observed muonium precession frequencies as a function of applied field (with an angle of $\sim 14^{\circ}$ between $B$ and $\langle 010\rangle$ ) at $T=25 \mathrm{~K}$. The lines are calculated values with $A_{X}=1.37, A_{Y}=6.67$, and $A_{Z}=11.52 \mathrm{MHz}$ with $(\alpha, \beta, \gamma)=\left(0^{\circ}, 57^{\circ}, 27^{\circ}\right)$ (solid) and $\left(0^{\circ}, 17^{\circ}, 0^{\circ}\right)$ (dashed).

angles are also consistent with the same $\mathrm{Mu}$ site. Our results are consistent with neutron diffraction results [44], infrared absorption experiments, and theoretical studies on hydrogen defect vibrational modes $[45,46]$ as well as other theoretical work [30]. However, they disagree with Refs. [47-50] which place the hydrogen on the O-O bond or the face of the cube between corner sharing $\mathrm{Sr}$ atoms and the $\mathrm{O}$ atoms at the face center. Note also that such sites, which have high symmetry, will result in an axially symmetric Mu hyperfine tensor. Surprisingly, we also find that the implanted muons occupy a different site from that occupied by other implanted impurities in STO such as Li [51,52].

In conclusion, we present a direct spectroscopic observation of a shallow hydrogenlike muonium state in STO. This confirms a theoretical prediction that interstitial hydrogen may act as a shallow donor in STO [28-30]. The formation of this muonium state appears below $\sim 70 \mathrm{~K}$ and implies an activation energy of $\sim 50 \mathrm{meV}$ in bulk which decreases to $\sim 25 \mathrm{meV}$ near the surface of the crystal. We find that the shallow impurity state has a fully anisotropic hyperfine tensor, with $A_{X}=1.4 \pm 0.1 \mathrm{MHz}, A_{Y}=6.7 \pm$ $0.1 \mathrm{MHz}$ and $A_{Z}=11.5 \pm 0.1 \mathrm{MHz}$. These results provide strong evidence of the sensitivity of the electronic properties of STO, and in particular its surface region, to impurities. Finally, since hydrogen is a ubiquitous impurity, these findings may prove crucial for interpretation of the variety of observed phenomena at LAO/STO interfaces [1-3]. We believe that hydrogen doping effect may be a possible explanation for the excess charge carriers at the interfaces of LAO/STO, and therefore require a more detailed experimental and theoretical consideration. We propose that a systematic study of the transport properties of LAO/STO interfaces as a function of hydrogen doping may provide quantitative information about this effect.

This work was performed at the Swiss Muon Source $(\mathrm{S} \mu \mathrm{S})$, Paul Scherrer Institute (PSI, Switzerland). We would like to thank Rob Kiefl, Kim Chow, and João Campos Gil for useful suggestions and fruitful discussions. We are also 
grateful to Ekaterina Pomjakushina for her assistance with the alignment of the crystals.

*zaher.salman@psi.ch

†thomas.prokscha@psi.ch

[1] A. Ohtomo and H. Y. Hwang, Nature (London) 427, 423 (2004).

[2] S. Thiel, G. Hammerl, A. Schmehl, C. W. Schneider, and J. Mannhart, Science 313, 1942 (2006).

[3] M. Huijben, G. Rijnders, D. H. A. Blank, S. Bals, S. V. Aert, J. Verbeeck, G. V. Tendeloo, A. Brinkman, and H. Hilgenkamp, Nat. Mater. 5, 556 (2006).

[4] A. Brinkman, M. Huijben, M. V. Zalk, J. Huijben, U. Zeitler, J. C. Maan, G. V. der Wiel, G. Rijnders, D. H. A. Blank, and H. Hilgenkamp, Nat. Mater. 6, 493 (2007).

[5] M. Ben Shalom, C. W. Tai, Y. Lereah, M. Sachs, E. Levy, D. Rakhmilevitch, A. Palevski, and Y. Dagan, Phys. Rev. B 80, 140403(R) (2009).

[6] Z. Salman et al., Phys. Rev. Lett. 109, 257207 (2012).

[7] N. Reyren et al., Science 317, 1196 (2007).

[8] R. Pentcheva and W. E. Pickett, Phys. Rev. B 74, 035112 (2006).

[9] M. S. Park, S. H. Rhim, and A. J. Freeman, Phys. Rev. B 74, 205416 (2006).

[10] M. Takizawa et al., Phys. Rev. Lett. 97, 057601 (2006).

[11] A. Kalabukhov, R. Gunnarsson, J. Borjesson, E. Olsson, T. Claeson, and D. Winkler, Phys. Rev. B 75, 121404 (2007).

[12] N. Nakagawa, H. Y. Hwang, and D. A. Muller, Nat. Mater. 5, 204 (2006).

[13] P. R. Willmott et al., Phys. Rev. Lett. 99, 155502 (2007).

[14] C. H. Ahn, J.-M. Triscone, and J. Mannhart, Nature (London) 424, 1015 (2003).

[15] S. Gemming and G. Seifert, Acta Mater. 54, 4299 (2006).

[16] D. R. Hamann, D. A. Muller, and H. Y. Hwang, Phys. Rev. B 73, 195403 (2006).

[17] J.-L. Maurice, C. Carrétéro, M.-J. Casanove, K. Bouzehouane, S. Guyard, E. Larquet, and J.-P. Contour, Phys. Status Solidi A 203, 2209 (2006).

[18] S. Okamoto, A. J. Millis, and N. A. Spaldin, Phys. Rev. Lett. 97, 056802 (2006).

[19] T. Higuchi, T. Tsukamoto, K. Kobayashi, S. Yamaguchi, Y. Ishiwata, N. Sata, K. Hiramoto, M. Ishigame, and S. Shin, Phys. Rev. B 65, 033201 (2001).

[20] T. Higuchi, T. Tsukamoto, N. Sata, M. Ishigame, Y. Tezuka, and S. Shin, Phys. Rev. B 57, 6978 (1998).

[21] H. Yukawa, K. Nakatsuka, and M. Morinaga, Solid State Ionics 116, 89 (1999).

[22] W.-j. Son, E. Cho, J. Lee, and S. Han, J. Phys. Condens. Matter 22, 315501 (2010).

[23] C. G. Van de Walle and J. Neugebauer, Nature (London) 423, 626 (2003).

[24] R. L. Lichti, K. H. Chow, and S. F. J. Cox, Phys. Rev. Lett. 101, 136403 (2008).
[25] S. F. J. Cox et al., Phys. Rev. Lett. 86, 2601 (2001).

[26] D. M. Hofmann, A. Hofstaetter, F. Leiter, H. Zhou, F. Henecker, B. K. Meyer, S. B. Orlinskii, J. Schmidt, and P. G. Baranov, Phys. Rev. Lett. 88, 045504 (2002).

[27] E. A. Davis, S. F. J. Cox, R. L. Lichti, and C. G. Van de Walle, Appl. Phys. Lett. 82, 592 (2003).

[28] K. Xiong, J. Robertson, and S. J. Clark, J. Appl. Phys. 102, 083710 (2007).

[29] P. W. Peacock and J. Robertson, Appl. Phys. Lett. 83, 2025 (2003).

[30] J. B. Varley, A. Janotti, and C. G. Van de Walle, Phys. Rev. B 89, 075202 (2014).

[31] S. F. J. Cox et al., J. Phys. Condens. Matter 18, 1079 (2006).

[32] B. D. Patterson, Rev. Mod. Phys. 60, 69 (1988).

[33] A. Yaouanc and P. D. de Réotier, Muon Spin Rotation, Relaxation, and Resonance: Applications to Condensed Matter (Oxford University Press, Oxford, 2010).

[34] E. Morenzoni et al., Physica (Amsterdam) 326B, 196 (2003).

[35] T. Prokscha, E. Morenzoni, K. Deiters, F. Foroughi, D. George, R. Kobler, A. Suter, and V. Vrankovic, Nucl. Instrum. Methods Phys. Res., Sect. A 595, 317 (2008).

[36] T. Prokscha, H. Luetkens, E. Morenzoni, G. J. Nieuwenhuys, A. Suter, M. Döbeli, M. Horisberger, and E. Pomjakushina, arXiv:1408.6972.

[37] T. Prokscha, E. Morenzoni, D. G. Eshchenko, N. Garifianov, H. Gluckler, R. Khasanov, H. Luetkens, and A. Suter, Phys. Rev. Lett. 98, 227401 (2007).

[38] K. A. Müller and H. Burkard, Phys. Rev. B 19, 3593 (1979).

[39] J. Ravichandran, W. Siemons, M. L. Scullin, S. Mukerjee, M. Huijben, J. E. Moore, A. Majumdar, and R. Ramesh, Phys. Rev. B 83, 035101 (2011).

[40] S. Hyun and K. Char, Appl. Phys. Lett. 79, 254 (2001).

[41] M. Senba, Phys. Rev. A 62, 042505 (2000).

[42] R. Cowley, Phil. Trans. R. Soc. A 354, 2799 (1996).

[43] S. F. J. Cox, Rep. Prog. Phys. 72, 116501 (2009).

[44] N. Sata, K. Hiramoto, M. Ishigame, S. Hosoya, N. Niimura, and S. Shin, Phys. Rev. B 54, 15795 (1996).

[45] M. C. Tarun and M. D. McCluskey, J. Appl. Phys. 109, 063706 (2011).

[46] J. T-Thienprasert, I. Fongkaew, D. J. Singh, M.-H. Du, and S. Limpijumnong, Phys. Rev. B 85, 125205 (2012).

[47] G. Weber, S. Kapphan, and M. Wöhlecke, Phys. Rev. B 34, 8406 (1986).

[48] D. Houde, Y. Lépine, C. Pépin, S. Jandl, and J. L. Brebner, Phys. Rev. B 35, 4948 (1987).

[49] S. Klauer and M. Wöhlecke, Phys. Rev. Lett. 68, 3212 (1992).

[50] L. Villamagua, R. Barreto, L. M. Prcel, and A. Stashans, Phys. Scr. 75, 374 (2007).

[51] Z. Salman, E. P. Reynard, W. A. MacFarlane, K. H. Chow, J. Chakhalian, S. R. Kreitzman, S. Daviel, C. D. P. Levy, R. Poutissou, and R. F. Kiefl, Phys. Rev. B 70, 104404 (2004).

[52] Z. Salman et al., Phys. Rev. Lett. 96, 147601 (2006). 